\title{
Study of microstructure in vanadium-palladium alloys by X-ray diffraction technique
}

\author{
J GHOSH*,‡, S K CHATTOPADHYAY, A K MEIKAP, S K CHATTERJEE and \\ P CHATTERJEE ${ }^{\dagger}$ \\ Department of Physics, National Institute of Technology, Durgapur 713 209, India \\ ${ }^{\dagger}$ Department of Physics, Vivekananda Mahavidyalaya, Haripal, Hoogly 712 405, India \\ ${ }^{\ddagger}$ Present Address: XRD Section, Central Glass and Ceramic Research Institute, Kolkata 700 032, India
}

MS received 8 March 2007

\begin{abstract}
Present study considers microstructural characterization of vanadium-based palladium (V-Pd) alloys, which are widely used in marine environment due to their high corrosion resistance. The $\mathrm{X}$-ray diffraction line profile analysis (XRDLPA) have been used to assess the microstructure in body centred cubic (bcc) V-Pd alloys having four different nominal compositions in wt.\%. X-ray diffraction line broadening analysis on V-Pd alloys has been performed by using different methods like the Warren-Averbach, double-Voigt and Rietveld methods. Finally microstructural defect parameters such as domain size $(D)$, r.m.s. microstrain $\left\langle\varepsilon^{2}\right\rangle^{1 / 2}$, twin fault $\left(\beta^{\prime}\right)$, spacing fault $(\alpha \varepsilon)$ and deformation stacking fault $(\alpha)$ were evaluated in these alloys by Fourier line shape analysis using Rietveld method in which the $X$-ray diffraction profiles of these alloys were described by the pseudo-Voigt function to fit the experimental data. From analysis it has been observed that twin fault, $\beta^{\prime}$, and the spacing fault, $\alpha \varepsilon$, are totally absent in these $b c c$ alloy systems because the twin fault, $\beta^{\prime}$, has been observed to be either negative or very small (within experimental error limit) for these alloy systems and the spacing fault, $\alpha \varepsilon$, appears to be negative. This analysis also revealed that the deformation stacking fault, $\alpha$, is significantly present in this alloy system and increases with Pd content.
\end{abstract}

Keywords. X-ray diffraction; metals and alloys; microstructure; Warren-Averbach method; double-Voigt method; Rietveld method.

\section{Introduction}

Vanadium-based alloys have been of much interest in recent years for their attractive properties which include low density, relatively high strength at elevated temperature, good fabricability and excellent corrosion resistance (Chung et al. 1996; Gan et al 2001; Evtikhin et al 2002; Ghosh et al 2005). Four compositions of bcc (Im3m, ICSD collection code: 44509) V-based Pd alloys in wt.\% as V-24\%Pd (alloy I), V-22\%Pd (alloy II), V-20\%Pd (alloy III) and $\mathrm{V}-18 \% \mathrm{Pd}$ (alloy IV) have been taken up for microstructure characterization by X-ray diffraction techniques of XRDLPA. Among various established methods for microstructural analysis from X-ray diffraction, Warren-Avebach $(1950,1952)$ method is highly elaborated and rigorous approach of size-strain analysis is used for evaluation of microstructural parameters like domain size $\langle D\rangle$ in $\AA$ and r.m.s. microstrain $\left\langle\varepsilon^{2}\right\rangle^{1 / 2}$ etc. The second approach is double-Voigt method (Balzar and Ledbetter 1993), in which both size and strain effects are modelled with Voigt function (convolution of the Cauchy

*Author for correspondence (jiten@ cgcri.res.in) and Gaussian functions). Third approach is the Rietveld (1969) method, a full-pattern fit method, which has been adopted in the present study for microstructure analysis of V-based Pd alloys. Rietveld method has some advantages over the other methods like whole fitting methodology for size-strain analysis, simultaneous refinement of crystal structure, microstructural parameters, capable of eliminating preferred orientation effect (if there is any) and so on. Considering all the benefits of the whole profile fitting methodology, the Rietveld's structure refinement procedure using LS1 software (Lutterotti and Scardi 1990) has been adopted in the present study for microstructure analysis of these alloys. Finally the twin fault parameter $\left(\beta^{\prime}\right)$, deformation stacking fault $(\alpha)$ and spacing fault $(\alpha \varepsilon)$ have been determined in the alloys by Fourier line shape analysis. The objective of this paper is to investigate how the microstructural parameters control the deformation characteristics of these alloy systems by $\mathrm{X}$-ray diffraction techniques.

\section{Experimental and methods of analysis}

The alloy ingots prepared by standard arc-melting method and homogenized at $800^{\circ} \mathrm{C}$ for $48 \mathrm{~h}$ and then cold work- 
ing on the alloys was done by hand filing using jeweler's file at room temperature. Then the powders were sieved through a 250 mesh screen after magnetically separating iron filing (if any), to produce flat diffractometer specimens for X-ray diffraction experiments. A specially prepared silicon $(\mathrm{Si})$ sample following the method reported by Van Berkum et al (Van Berkum 1994; Van Berkum et al 1995) was used as the standard sample to encompass the effect of instrumental broadening of the diffractometer. It was also assumed that $\mathrm{Si}$ standard specimen did not have any size and/or strain broadening. The X-ray diffraction profiles of cold-worked samples were recorded from $35^{\circ}$ to $100^{\circ}$ by Philips PW 1830 X-ray diffractometer. The diffractometer operating at $35 \mathrm{kV}$ and $20 \mathrm{~mA}$ operating in step scanning mode of $0.02^{\circ}$ in $2 \theta$ using Nifiltered $\mathrm{CuK}_{\alpha}$ radiation and counting time of $4 \mathrm{~s}$ per step. The diffraction profiles 110, 200, 211 and 220 were recorded for the alloys I to IV. Three different approaches for analysis viz. Warren-Averbach, double-Voigt and Rietveld methods were adopted for microstructure characterization in these alloy systems.

\subsection{Warren-Averbach method}

In this method, the domain size and microstrain were evaluated by expressing observed XRD profiles in Fourier series. The coefficients of this series include the effect of deformations and instrumental effects. The instrumental effects can be separated following Stoke's (1948) method i.e. by deconvoluting the Fourier coefficients of the Fourier series into which the profiles from standard silicon are expressed. These Stoke's corrected Fourier coefficients, $A_{L}$, are the product of two terms: the size $\left(A_{L}^{\mathrm{S}}\right)$ and the distortion $\left(A_{L}^{\mathrm{D}}\right)$ coefficients. The size coefficients $\left(A_{L}^{\mathrm{S}}\right)$ and distortion coefficients $\left(A_{L}^{\mathrm{D}}\right)$ can be separated from the intercept at $1 / d^{2}=0$ of the plot of $\ln A_{L}$ vs $1 / d^{2}$, where $d$ is the interplanar spacing. The micro-strain $\left\langle\varepsilon^{2}(L)\right\rangle$ can be determined from distortion coefficient, $A_{L}^{\mathrm{D}}$, by assuming Gaussian strain distribution as (Warren 1969)

$$
A_{L}^{\mathrm{D}}=\exp \left[-2 \pi^{2}<\varepsilon^{2}(L)>L^{2} / d^{2}\right] .
$$

Then the domain size was estimated from the size coefficients, $A_{L}^{\mathrm{S}}$, for multiple order reflections (110) and (200).

\subsection{Double-Voigt method}

In this method, both size and strain broadened profiles are assumed to be Voigtian. The Fourier coefficients in terms of a distance, $L$, perpendicular to the diffracting planes is obtained by Fourier transform of a Voigt function and can be written as

$$
A_{L}=\exp \left[-2 L \beta_{\mathrm{C}}-\pi L^{2} \beta_{\mathrm{G}}^{2}\right],
$$

where $\beta_{\mathrm{C}}$ and $\beta_{\mathrm{G}}$ are the Cauchy and Gaussian components of the integral breadth, $\beta$, respectively. These two components in (2) follow the convolution principle and the following relations relate them

$$
\begin{aligned}
& \beta_{\mathrm{C}}=\beta_{\mathrm{SC}}+\beta_{\mathrm{DC}}, \\
& \beta_{\mathrm{G}}^{2}=\beta_{\mathrm{SG}}^{2}+\beta_{\mathrm{DG}}^{2},
\end{aligned}
$$

where $\beta_{\mathrm{SC}}$ and $\beta_{\mathrm{DC}}$ are the Cauchy components of size and strain in integral breadths, respectively and $\beta_{\mathrm{SG}}$ and $\beta_{\mathrm{DG}}$ are the corresponding Gaussian components. The surface weighted domain size and mean-square strain were estimated from multiple-order reflections (110) and (220) by the following relations:

$$
\begin{aligned}
& -\left(\frac{d A_{\mathrm{L}}^{\mathrm{S}}}{d L}\right)_{L \rightarrow 0}=\frac{1}{\langle D\rangle_{S}}=\left(2 \beta_{\mathrm{SC}}\right), \\
& \left\langle\varepsilon^{2}(L)\right\rangle=\frac{1}{s^{2}}\left(\frac{\beta_{\mathrm{DG}}^{2}}{2 \pi}+\frac{\beta_{\mathrm{DC}}}{\pi^{2} L}\right),
\end{aligned}
$$

where $s=2 \sin \theta / \lambda$.

\subsection{Rietveld method}

The microstructural parameters like average domain size and r.m.s. microstrain were also estimated from X-ray diffraction by Rietveld method using LS1 program. This program is based on the Rietveld method combined with the Fourier analysis of the broadened peaks. The powder diffraction patterns were simulated with the help of this program providing all necessary structural, instrumental information and some starting values of microstructural parameters of individual phases. In the present study, it was assumed that the full width at half maximum (FWHM) of standard $\mathrm{Si}$ reflections followed the Caglioti relation (Caglioti et al 1958):

$$
\mathrm{FWHM}^{2}=U \tan ^{2} \theta+V \tan \theta+W .
$$

The $U, V, W$ parameters of instrumental broadening, instrumental asymmetry and Gaussianity of reflections were evaluated for the present setting of the instrument using this standard Si. All instrumental parameters were incorporated in the convoluting fitting process and the values were kept fixed for estimation of values of all these parameters solely for a sample under investigation. Then effective domain size and r.m.s. microstrain were evaluated from best fitting parameters.

2.3a Line shape analysis: The effective domain size $\left(D_{\mathrm{e}}\right)$ is related to the average domain size $(\langle D\rangle)$ and the faults (deformation fault, $\alpha$ and twin fault, $\beta^{\prime}$ ) by the following relation (Warren 1969)

$$
\frac{1}{D_{\mathrm{e}}}=\frac{1}{\langle D\rangle}+\left[\left(1 \cdot 5 \alpha+\beta^{\prime}\right) / a\right]\left[\sum_{b}\left|L_{0}\right| /(u+b) h_{0}\right] .
$$


where $h_{0}=\left(h^{2}+k^{2}+l^{2}\right)^{1 / 2}, u$ is the unbroadened component by faulting for $L_{0}=3 N$ reflections, $b$ the broadened component by faulting for $L_{0}=3 N \pm 1$ reflections and $\sum\left|L_{0}\right| /(u+b) h_{0}=0.471$ for 110 reflection and 1.333 for 200 reflection. The faulting parameter $\left(1 \cdot 5 \alpha+\beta^{\prime \prime}\right)$ can be separated and evaluated from the two reflections, 110 and 200 , assuming the average domain size $\langle D\rangle$ to remain uniform.

2.3b Line asymmetry analysis: The evaluation of twin fault, $\beta^{\prime}$, from the peak asymmetry analysis is theoretically possible for $b c c$ structure but the effect of this in peak asymmetry is so small that no meaningful evaluation of it is possible from the shift of the centre of gravity $(\mathrm{CG})$ of the profiles from their peak maxima (PM). However, the shift of $\mathrm{CG}$ of the profiles calculated by using a suitable program, from peak maximum is

$$
\left(2 \theta_{\mathrm{CG}}^{0}-2 \theta_{\mathrm{PM}}^{0}\right)_{h k l}=-14 \cdot 6 X_{h k l} \tan \theta \cdot \beta^{\prime},
$$

where $\theta$ is the Bragg's angle of the peak and $X_{h k l}$ a constant and the value was calculated to be 0.17 for (110) reflection, -0.33 for (200) reflection and 0.25 for (211) reflection (Wagner 1956; Warren 1969; Chatterjee 1999).

2.3c Evaluation of spacing fault $(\alpha \varepsilon)$ : peak shift analysis: Actually for $b c c$ metals, no peak shift for the presence of deformation fault, $\alpha$, can be observed practically, however, there may be some shift in the peak maximum due to the change in spacing in the faulted region (Langford 1992; Balzar and Ledbetter 1993; Balzar and Popovie 1996). This change in spacing known as spacing fault $(\alpha \varepsilon)$ can be evaluated from peak shift analysis, though the shift due to this effect is practically very small. Now if $\alpha$ is the deformation stacking fault, and $\varepsilon$ the change in spacing in faulted plane (spacing fault), then $\alpha \varepsilon$ can be written as layer fault, which contributes in the shift of the peak (Wagner 1956). Now assuming the long range residual stress $(\sigma)$ to be zero in powder sample because of perfect randomness, the relative peak shift, $\delta\left(\Delta 2 \theta^{0}\right)_{h k l-h^{\prime} k^{\prime} l^{\prime}}$, can be written as

$$
\delta\left(\Delta 2 \theta^{0}\right)_{h k l-h^{\prime} k^{\prime} l^{\prime}}=A_{h k l-h^{\prime} k^{\prime} l^{\prime}}(\Delta a / a)+H_{h k l-h^{\prime} k^{\prime} l^{\prime}}(\alpha \varepsilon),
$$

where, $h k l$ and $h^{\prime} k^{\prime} l^{\prime}$ are two neighbouring reflections and $A_{h k l-h^{\prime} k^{\prime} l^{\prime}}, H_{h k l-h^{\prime} k^{\prime} l^{\prime}}$ are given as

$$
\begin{aligned}
& A_{h k l-h^{\prime} k^{\prime} l^{\prime}}=(-360 / \pi)\left(\tan \theta_{h k l}-\tan \theta_{h^{\prime} k^{\prime} l^{\prime}}\right), \\
& H_{h k l-h^{\prime} k^{\prime} l^{\prime}}=(-360 / \pi)\left(J_{h k l} \tan \theta_{h k l}-J_{h^{\prime} k^{\prime} l^{\prime}} \tan \theta_{h^{\prime} k^{\prime} l^{\prime}}\right),
\end{aligned}
$$

where $J_{h k l}$ is a faulting coefficient for $b c c$ structure and for (110), (200) neighbouring reflections, the values were calculated to be $0 \cdot 21$ for (110) and $-0 \cdot 17$ for (200).

\section{Results and discussion}

The nominal compositions of the alloys used in the present study are shown in table 1. Four different compositions (alloys I-IV) of V-Pd alloys were used to investigate how the microstructural parameter varies with Pd content in these alloys. Figure 1 shows the X-ray diffraction patterns from $b c c$ V-Pd alloys (alloys I-IV). Different X-ray diffraction line profile analysis methods were applied on the diffraction profile of this alloy for microstructure characterization. In the fitting process, we have employed a pattern decomposition algorithm using different profile functions numerically convoluted with a truncated exponential function to evaluate the profile shape parameters like the integral breadths $(\beta)$ and full width at half maximum (FWHM) of different reflections. Lattice parameters have been calculated from the peak position $\left(2 \theta_{0}\right)$ for all observable reflections using Cohen's least-squares method (Cullity 1978) and the values are shown in table 1. It is observed that the lattice parameter increases almost systematically with Pd content and is shown in figure $2 \mathrm{a}$. This is obvious from the size difference of $\mathrm{V}$ and $\mathrm{Pd}$ atoms. In the fitting process, the Lorentzian content, $\eta$, Gaussian content $(1-\eta)$, half width at half maximum, $\omega$, Bragg $2 \theta$ position and the peak height, $I_{0}$, were simultaneously re-

\begin{tabular}{|c|c|c|c|c|c|}
\hline Alloy & $\begin{array}{l}\text { Nominal compositions } \\
\text { (wt.\%) }\end{array}$ & $\begin{array}{l}\text { Cell parameter, } a(\AA) \\
\text { Error limit, } \pm 0.0004 \AA\end{array}$ & $h k l$ & $\beta_{\mathrm{C}}($ degree $)$ & $\beta_{\mathrm{G}}($ degree $)$ \\
\hline \multirow[t]{2}{*}{ Alloy I } & $\mathrm{V}, 76 \% ; \mathrm{Pd}, 24 \%$ & 3.0399 & 110 & 0.46591 & $0 \cdot 24267$ \\
\hline & & & 220 & 0.67997 & $0 \cdot 74214$ \\
\hline \multirow[t]{2}{*}{ Alloy II } & $\mathrm{V}, 78 \% ; \mathrm{Pd}, 22 \%$ & 3.0383 & 110 & 0.46637 & $0 \cdot 30974$ \\
\hline & & & 220 & 0.66314 & $0 \cdot 71937$ \\
\hline \multirow[t]{2}{*}{ Alloy II } & $\mathrm{V}, 80 \% ; \mathrm{Pd}, 20 \%$ & $3 \cdot 0397$ & 110 & $0 \cdot 34730$ & $0 \cdot 28027$ \\
\hline & & & 220 & $0 \cdot 22079$ & $0 \cdot 21377$ \\
\hline \multirow[t]{2}{*}{ Alloy IV } & $\mathrm{V}, 82 \% ; \mathrm{Pd}, 18 \%$ & $3 \cdot 0349$ & 110 & $1 \cdot 32626$ & $0 \cdot 28001$ \\
\hline & & & 220 & $0 \cdot 87187$ & 0.42123 \\
\hline
\end{tabular}
fined along with the parameters of the linearly varying

Table 1. Values of lattice parameter $(a)$, Cauchy component $\left(\beta_{\mathrm{C}}\right)$ and Gaussian component $\left(\beta_{\mathrm{G}}\right)$ of integral breadth, for V-Pd alloys along with alloy composition. 

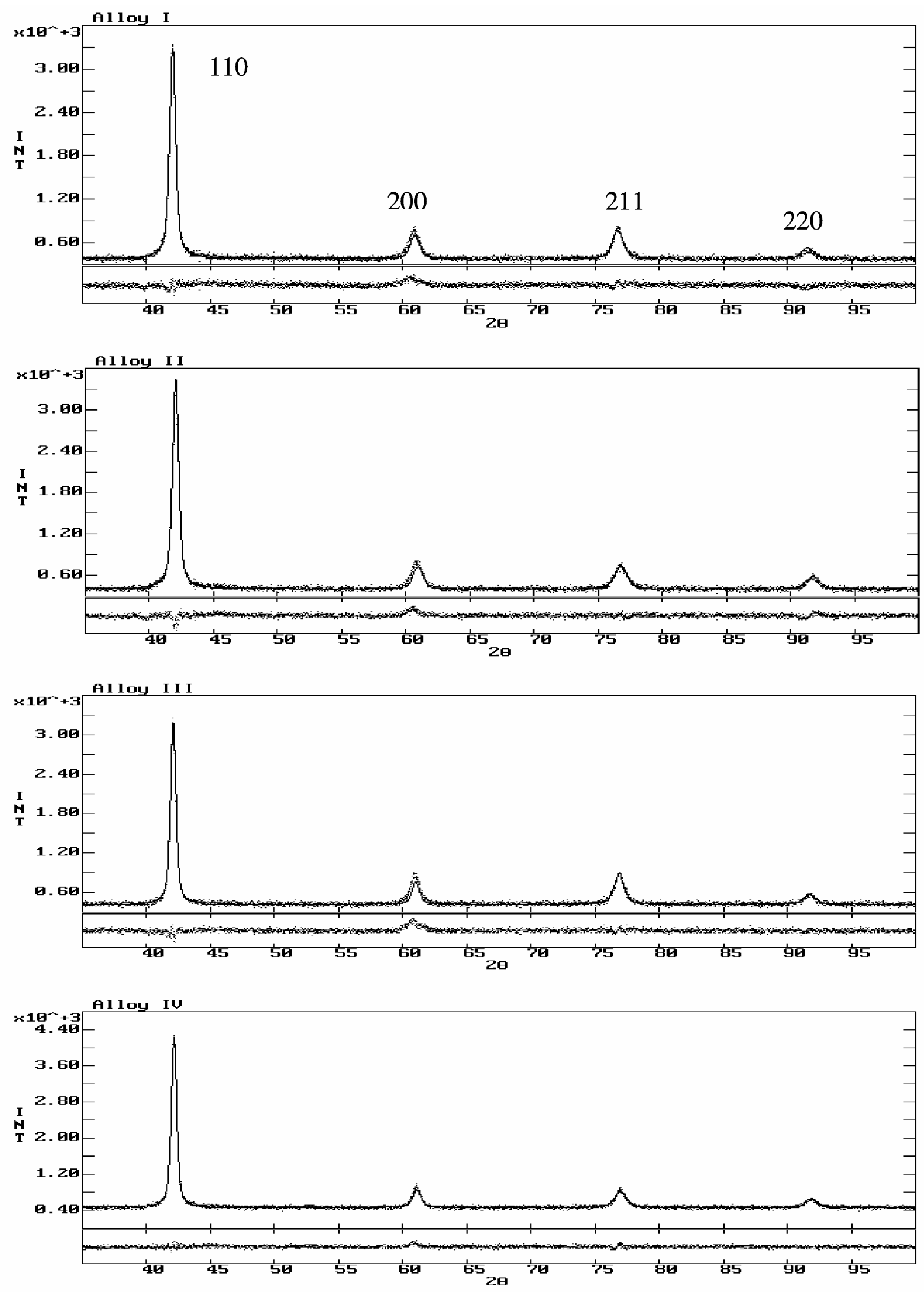

Figure 1. X-ray diffraction patterns of cold-worked powders of V-Pd alloys I-IV. The respective residual is drawn in the same scale at the bottom of each pattern. 

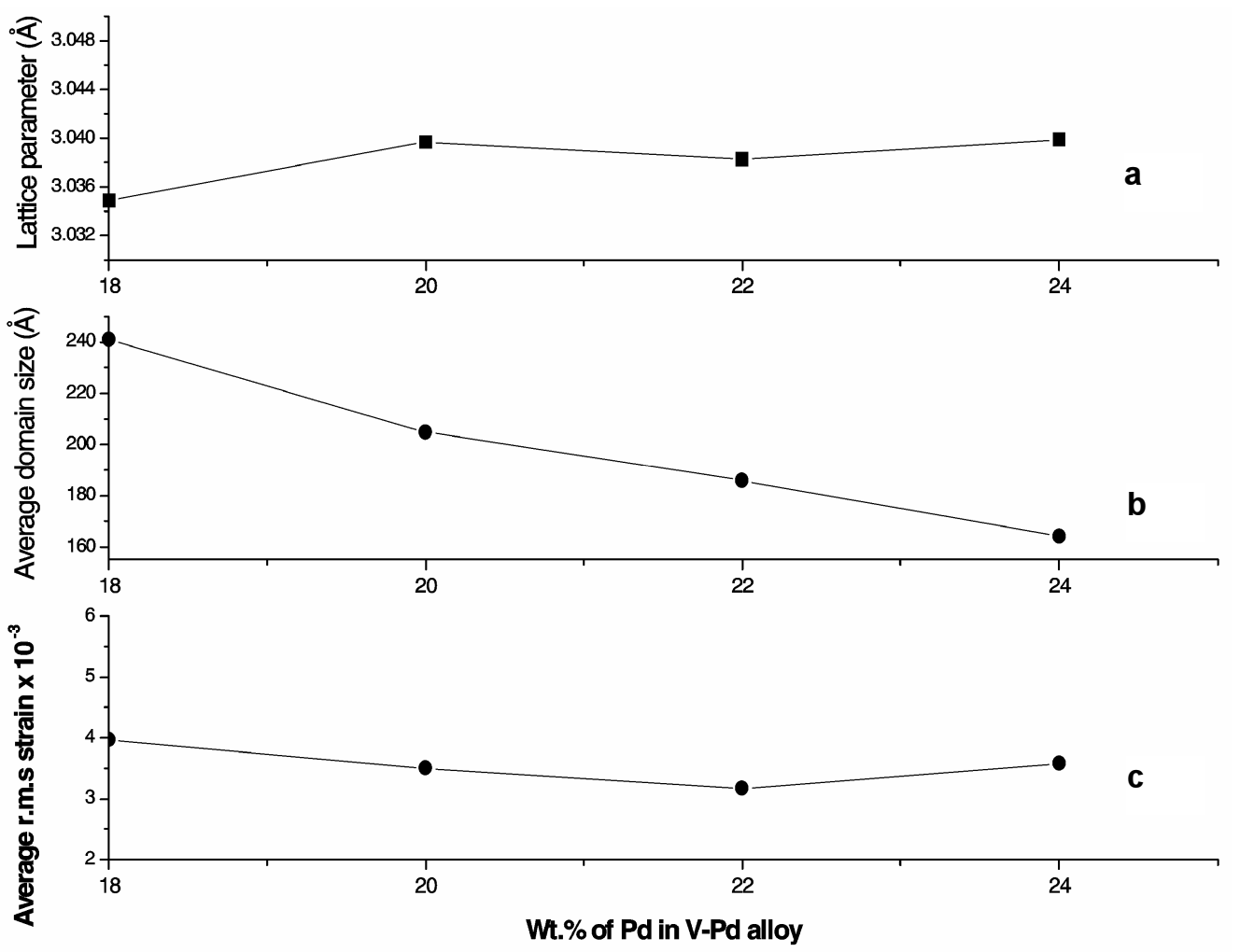

Figure 2. a. Variation of lattice parameter $(a)$ with Pd content in V-Pd alloy, b. variation of average domain size $(D)$ with Pd content in V-Pd alloy and c. variation of average r.m.s. strain with Pd content in V-Pd alloy.

Table 2. Values of Cauchy and Gaussian component of size and strain along [110] direction from Voigt function.

\begin{tabular}{lcccc}
\hline Alloy & $\beta_{\mathrm{DC}}\left(10^{3}\right)$ & $\beta_{\mathrm{SC}}\left(10^{3}\right)$ & $\beta_{\mathrm{SG}}\left(10^{3}\right)$ & $\beta_{\mathrm{DG}}\left(10^{3}\right)$ \\
\hline Alloy I & 0.02 & 4.78 & Negative & 3.05 \\
Alloy II & 0.10 & 4.83 & 1.89 & 2.68 \\
Alloy III & 1.99 & 1.68 & 3.28 & Negative \\
Alloy IV & 1.15 & 2.31 & 2.83 & 0.87 \\
\hline
\end{tabular}

background. Then the Gaussian and Cauchy components of the integral breadths $\left(\beta_{\mathrm{C}}, \beta_{\mathrm{G}}\right)$ of the true broadened profile of the specimen obtained after deconvolution (Keijser et al 1982) are given in table 1. Assuming both the size and the strain broadened profiles to be approximated by Voigt function, the Cauchy and Gaussian components $\left(\beta_{\mathrm{SC}}, \beta_{\mathrm{SG}}, \beta_{\mathrm{DC}}\right.$ and $\left.\beta_{\mathrm{DG}}\right)$ of the size and strain broadened profiles were separated as described in $\$ 2.2$ and the values are given in table 2 . From table 2 , it is observed, in general, that both size and strain broadened profiles have Cauchy and Gaussian contributions. Whenever the true profiles are of Cauchy type, the Gaussian components are set to be zero. $\beta_{\mathrm{SG}}$ has a negative value for alloy I which indicates that the size broadened profile is purely Cauchy type. Similarly negative value of $\beta_{\mathrm{DC}}$ indicates that the strain broadened profile is purely Gaussian type. Strain broadened profile is purely Cauchy type in alloy III and size broadened profile is purely Cauchy type in alloy I. In general, both size and strain broadened profiles have Cauchy and Gaussian contributions. In double-Voigt method, the Fourier coefficients, $A_{L}$, were determined by (2) and Warren-Averbach method after assuming the observed diffraction profiles to be represented by a Fourier series. In Warren-Averbach method, from the $A_{L}$ coefficients determined for various ranges $\left(\theta_{1}\right.$ and $\left.\theta_{2}\right)$ of the profiles, the best possible coefficients with minimum fluctuation were taken. Then domain size and microstrain were estimated for alloys I-IV from $A_{L}^{\mathrm{S}}$ and $A_{L}^{\mathrm{D}}$ coefficients and the values are shown in table 3 . From table 3 , it has been observed that domain and microstrain both decrease with Pd content. In Warren-Averbach method it was assumed that the strain would follow Gaussian nature. But from table 2, it has been seen that both Gaussian and Cauchy component significantly contribute to the strain. Hence, the values of domain size and microstrain are some times overestimated due to underestimated Cauchy component of the profile. It has also been seen from table 2 that the strain profile is purely Cauchy type $\left(\beta_{\mathrm{DG}}=0\right)$ for alloy III. Hence, the values of size and strain both were overestimated for alloy III. In practice, 


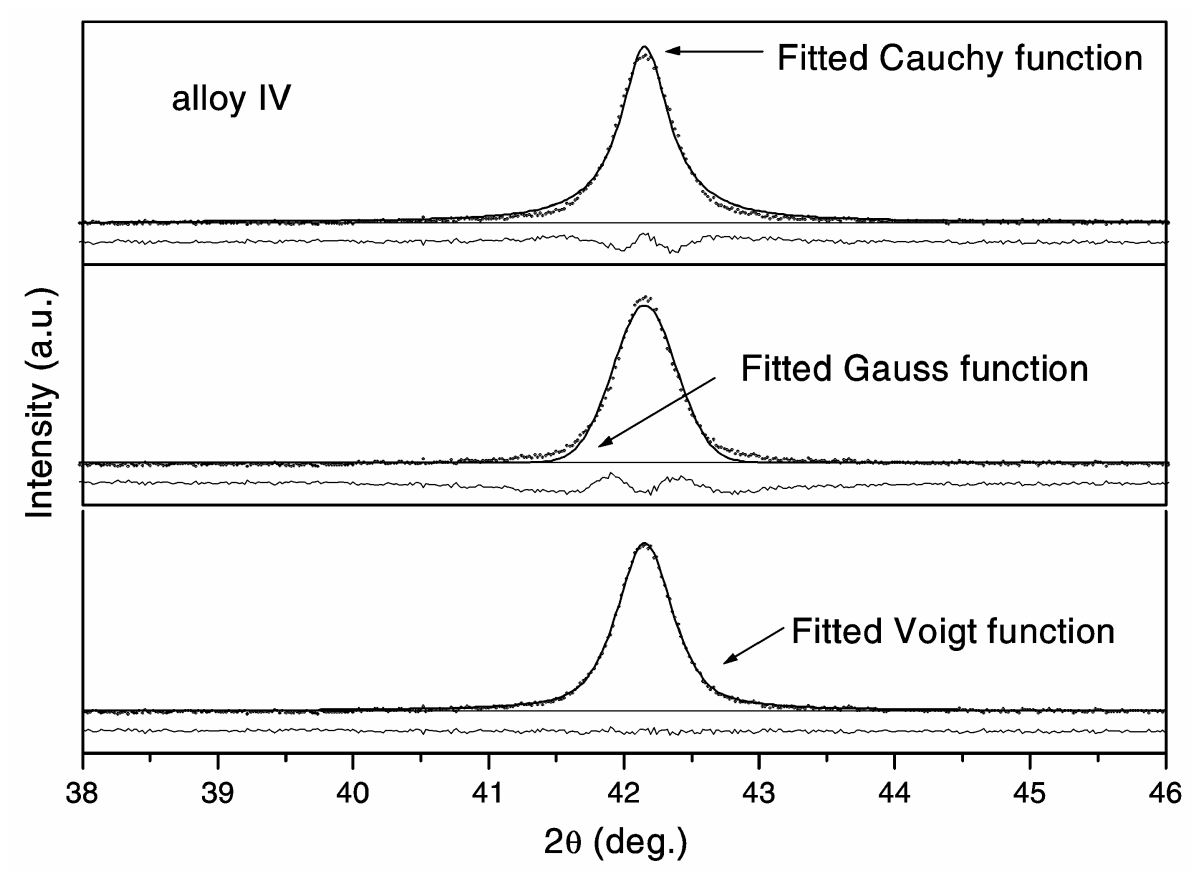

Figure 3. Cauchy, Gauss and Voigt-function fits to the physically broadened 110 line profile of alloy IV. Difference patterns plotted in the same scale at the bottom of each pattern

Table 3. Comparison of the defect structure parameters determined by X-ray diffraction for different approaches along (110) direction.

\begin{tabular}{lccccc}
\hline & \multicolumn{2}{c}{ Warren-Averbach } & & \multicolumn{2}{c}{ Double-Voigt } \\
\cline { 2 - 3 } \cline { 5 - 6 } Alloy & $\langle D\rangle_{\mathrm{S}}(\AA)$ & $\left\langle\varepsilon^{2}\right\rangle^{1 / 2}\left(\times 10^{3}\right)$ & & $\langle D\rangle_{\mathrm{S}}(\AA)$ & $\left\langle\varepsilon^{2}\right\rangle^{1 / 2}\left(\times 10^{3}\right)$ \\
\hline Alloy I & 108.47 & 2.85 & & 104.58 & 2.86 \\
Alloy II & 103.68 & 2.48 & & 103.45 & 2.49 \\
Alloy III & 240.14 & $4 \cdot 16$ & & 297.85 & 4.32 \\
Alloy IV & 193.67 & 3.34 & & 216.88 & 3.35 \\
\hline
\end{tabular}

Error limit for domain size $\pm 5 \AA$; error limit for r.m.s. strain: $\pm 0.03 \times 10^{-3}$.

Gaussian and Cauchy components both significantly contribute to the size and strain broadened profiles.

It has been observed that Voigt function satisfactorily fits the physically broadened line profiles. From figure 3, it has also been observed from different plots that the Voigt function shows a superior and overall satisfactory fit for this alloy system. From this analysis, it has been observed that Cauchy function fits fairly well with the tails of the profile, whereas peak of the profile do not exactly fit with this function. On the contrary, peak of the profile fits fairly with Gauss function but tails fall off too rapidly. This was also observed by Davor Balzar in his work (Balzar 1999).

From double-Voigt method, the average surfaceweighted domain size, calculated for alloys I-IV by (5) and r.m.s. strain at $L=50 \AA$ by (6), are shown in table 3 along with the data available from the Warren-Averbach analysis. The domain size, microstrain and faulting probabilities were determined by the Rietveld method using pseudo-Voigt function. The domain size and microstrain were determined from the best fitting parameters and the values are shown in table 6 . The quality of fitting was assessed from various numerical criteria of fit, viz. the profile residual factor $\left(R_{\mathrm{b}}\right)$, the weighted residual factor $\left(R_{\mathrm{wp}}\right)$, the expected residual factor $\left(R_{\exp }\right)$ and the goodness of fit (GOF), as obtained from the Rietveld analysis using LS1 program, all revealing good fitting. The values of these various reliability parameters as obtained from Rietveld analysis of these alloy systems are shown in table 5. The quality of the fittings of observed diffraction patterns with the simulated patterns are shown by residual plots in figure 1 . From table 6 , it has been observed that average domain size systematically decreases with $\mathrm{Pd}$ content in this alloy system while average r.m.s. microstrain remained almost constant in this system (figures $2 \mathrm{~b}, \mathrm{c})$.

Peak maximum (PM) ( $2 \theta$ in degree) and centre of gravity $(\mathrm{CG})$ ( $2 \theta$ in degree) of observed XRD profile of the alloys were estimated using pseudo-Voigt function and the values are shown in table 4 . Then twin fault density $\left(\beta^{\prime}\right)$ was evaluated by line asymmetry analysis using (8) for (110), (200) and (211) reflections from the PM and CG of observed diffraction profile. The faulting parameter $\left(1.5 \alpha+\beta^{\prime}\right)$ was evaluated from two reflections (110) and 


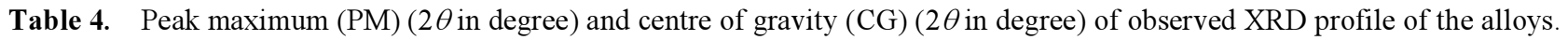

\begin{tabular}{|c|c|c|c|c|c|c|c|c|c|}
\hline \multirow[b]{2}{*}{ Alloy } & \multicolumn{3}{|c|}{$\begin{array}{c}\text { Peak maximum }(\mathrm{PM}) \\
2 \theta(\text { degree })\end{array}$} & \multicolumn{3}{|c|}{$\begin{array}{l}\text { Centre of gravity }(\mathrm{CG}) 2 \theta \text { (degree) } \\
\text { Error limit, } \pm 0 \cdot 003 \text { (degree) }\end{array}$} & \multicolumn{3}{|c|}{$\left(2 \theta_{\mathrm{CG}}^{0}-2 \theta_{\mathrm{PM}}^{0}\right)$} \\
\hline & 110 & 200 & 211 & 110 & 200 & 211 & 110 & 200 & 211 \\
\hline Alloy I & $42 \cdot 02$ & 61.00 & $76 \cdot 66$ & $42 \cdot 05$ & $60 \cdot 84$ & $76 \cdot 73$ & 0.03 & $-0 \cdot 16$ & 0.07 \\
\hline Alloy II & $42 \cdot 08$ & $60 \cdot 74$ & $76 \cdot 88$ & $42 \cdot 13$ & $60 \cdot 91$ & $76 \cdot 80$ & 0.05 & $0 \cdot 17$ & -0.08 \\
\hline Alloy III & $42 \cdot 08$ & $60 \cdot 86$ & $76 \cdot 94$ & $42 \cdot 14$ & $60 \cdot 94$ & $76 \cdot 95$ & 0.06 & $0 \cdot 08$ & 0.01 \\
\hline Alloy IV & $42 \cdot 16$ & $61 \cdot 12$ & $76 \cdot 9$ & $42 \cdot 09$ & $61 \cdot 21$ & $76 \cdot 99$ & _-0.07 & $0 \cdot 09$ & 0.09 \\
\hline
\end{tabular}

Table 5. The values of lattice parameters and various reliability parameters as obtained from Rietveld analysis.

\begin{tabular}{lccccc}
\hline & & & & \multicolumn{2}{c}{$\begin{array}{c}\text { Lattice parameters }(\AA) \\
\text { Error limit: } \pm 0.0002(\AA) \\
\text { Alloy }\end{array}$} \\
\hline Alloy I & $R_{\mathrm{b}}$ & $R_{\mathrm{wp}}$ & $R_{\exp }$ & GOF & $a$ \\
Alloy II & 5.242 & 5.589 & 4.842 & 1.154 & 3.0435 \\
Alloy III & 5.019 & 5.253 & 4.696 & 1.119 & 3.0441 \\
Alloy IV & 5.406 & 5.467 & 4.650 & 1.176 & 3.0430 \\
\hline
\end{tabular}

Table 6. Values of size-strain of the V-Pd alloys as obtained from Rietveld analysis.

\begin{tabular}{|c|c|c|c|c|c|}
\hline \multirow[b]{2}{*}{ Alloy } & \multirow{2}{*}{$\begin{array}{l}\text { Average domain size, } D(\AA) \\
\quad \text { Error limit, } \pm 5 \AA\end{array}$} & \multirow{2}{*}{$\begin{array}{l}\text { Average r.m.s. strain } \\
\quad\left\langle\left\langle\varepsilon^{2}(L)^{1 / 2}\right\rangle\left(10^{-3}\right)\right. \\
\text { Error limit, } \pm 0.03 \times 10^{-3}\end{array}$} & \multicolumn{3}{|c|}{$D_{\mathrm{e}}(\AA)$ Error limit, $\pm 5 \AA$} \\
\hline & & & 110 & 200 & 211 \\
\hline Alloy I & 164 & $3 \cdot 58$ & 103 & 45 & 89 \\
\hline Alloy II & 186 & $3 \cdot 17$ & 97 & 56 & 78 \\
\hline Alloy III & 205 & $3 \cdot 50$ & 127 & 80 & 103 \\
\hline Alloy IV & 241 & $3 \cdot 97$ & 142 & 98 & 128 \\
\hline
\end{tabular}

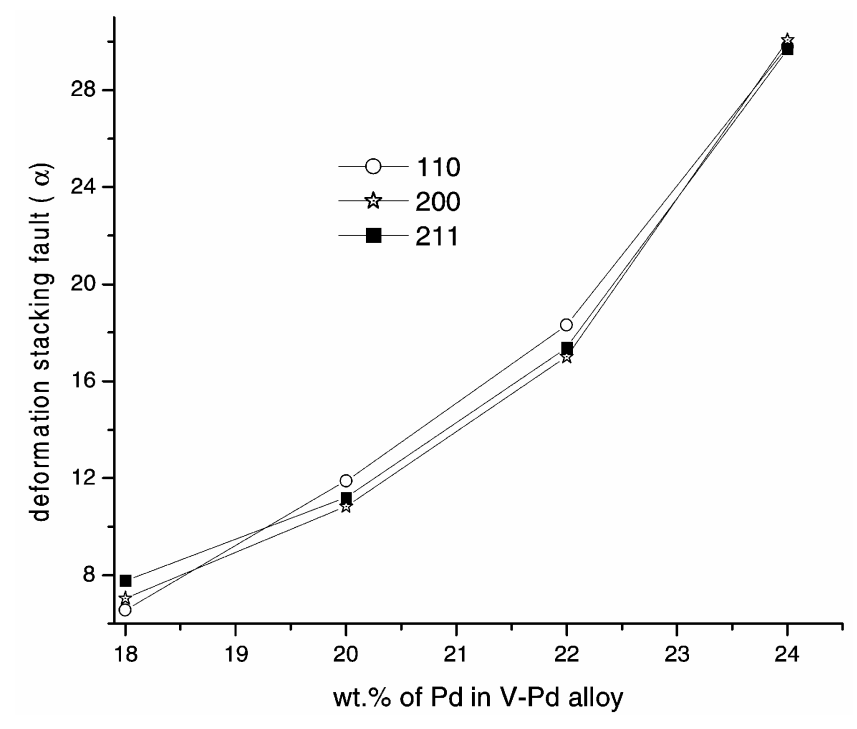

Figure 4. Variation of deformation stacking fault $(\alpha)$ with $\mathrm{Pd}$ content in V-Pd alloy.

(200) by assuming the average domain size $\langle D\rangle$ to remain constant. The values of twin fault parameter, $\beta^{\prime}$, deformation stacking fault, $\alpha$, calculated from the value of the faulting parameter $\left(1.5 \alpha+\beta^{\prime}\right)$ and spacing fault $\alpha \varepsilon$ are also shown in table 7 along with their error limits. The twin fault parameter $\left(\beta^{\prime}\right)$ has been observed to be either negligibly small or negative. This shows that twin faults are absent in this $b c c$ system. The negative values of the spacing fault $(\alpha \varepsilon)$ confirmed the absence of the spacing faults in the system. The deformation stacking fault, $\alpha$, obtained from this analysis is however, not negligible. From figure 4 , it has been observed that the deformation stacking fault $(\alpha)$ significantly increases with $\mathrm{Pd}$ content in this alloy system. It can also be concluded that these vanadium alloys show the presence of significant stacking fault density.

\section{Conclusions}

Size and strain parameters were calculated from three different approaches like double-Voigt, Warren-Averbach and Rietveld method by XRDLPA. This analysis reveals that the average domain size systematically decreases with $\mathrm{Pd}$ content in this alloy system while average r.m.s. microstrain remained almost constant in the system. It has been observed from XRDLPA by Rietveld method that the twin fault, $\beta^{\prime}$, and spacing fault, $\alpha \varepsilon$, are totally 
Table 7. Values of compound fault parameter $\left(1 \cdot 5 \alpha+\beta^{\prime}\right)$ and growth fault parameter, $\beta^{\prime}$, deformation stacking fault, $\alpha$, and the spacing fault $(\alpha \varepsilon)$ from Rietveld analysis.

\begin{tabular}{|c|c|c|c|c|c|c|c|c|}
\hline \multirow[b]{2}{*}{ Alloy } & \multirow{2}{*}{$\begin{array}{l}\left(1.5 \alpha+\beta^{\prime}\right)\left(\times 10^{3}\right) \\
\text { Error limit, } \pm 0.003\end{array}$} & \multicolumn{3}{|c|}{$\begin{array}{c}\beta^{\prime}\left(\times 10^{3}\right) \\
\text { Error limit, } \pm 3\end{array}$} & \multicolumn{3}{|c|}{$\begin{array}{c}\alpha\left(\times 10^{3}\right) \\
\text { Error limit, } \pm 3\end{array}$} & \multirow[b]{2}{*}{$\alpha \varepsilon\left(\times 10^{3}\right)$} \\
\hline & & 110 & 200 & 211 & 110 & 200 & 211 & \\
\hline Alloy I & $44 \cdot 13$ & $-0 \cdot 55$ & $-0 \cdot 98$ & -0.42 & $29 \cdot 79$ & $30 \cdot 07$ & $29 \cdot 70$ & $-7 \cdot 72$ \\
\hline Alloy II & $26 \cdot 57$ & $-0 \cdot 91$ & 1.05 & 0.48 & $18 \cdot 32$ & $17 \cdot 01$ & $17 \cdot 39$ & $-23 \cdot 82$ \\
\hline Alloy III & $16 \cdot 75$ & $-1 \cdot 10$ & 0.49 & $-0 \cdot 06$ & $11 \cdot 90$ & $10 \cdot 84$ & $11 \cdot 21$ & $-17 \cdot 47$ \\
\hline Alloy IV & $11 \cdot 13$ & $1 \cdot 28$ & $0 \cdot 55$ & $-0 \cdot 54$ & $6 \cdot 57$ & $7 \cdot 05$ & $7 \cdot 78$ & $-10 \cdot 51$ \\
\hline
\end{tabular}

absent in this alloy while the deformation stacking fault, $\alpha$, has significant presence and shows an increasing trend with the increase of Pd content. This vanadium-based alloy system can be used as structural materials in corrosive atmosphere, as vanadium shows corrosion resistance because of the significant presence of deformation stacking fault.

\section{References}

Balzar D 1999 Voigt-function model in diffraction linebroadening analysis, defects and microstructure: Analysis by diffraction (eds) R L Snyder et al (New York: Oxford University Press) p. 94

Balzar D and Ledbetter H 1993 J. Appl. Crystallogr. 2697

Balzar D and Popovie S 1996 J. Appl. Crystallogr. 2916

Caglioti G, Paoletti A and Ricci F P 1958 Nucl. Instrum. Meth. 35223

Cullity B D 1978 Elements of X-ray diffraction (Reading, MA: Addison-Wesley Publishing Co.) p. 363

Chung H M et al 1996 J. Nucl. Mater. 239139
Chatterjee S K 1999 X-ray diffraction: Its theory and applications (New Delhi: Prentice Hall of India)

Evtikhin V A et al 2002 J. Nucl. Mater. 307-311 591

Gan Y X et al 2001 J. Nucl. Mater. 299157

Ghosh J et al 2005 Jpn J. Appl. Phys. 446678

Keijser Th H et al 1982 J. Appl. Crystallogr. 15308

Langford J I 1992 Accuracy in powder diffraction II, NIST special publication no. 846 (eds) E Prince and J K Stalick (Washington DC: US Department of Commerce) p. 110

Lutterotti L and Scardi P 1990 J. Appl. Crystallogr. 23246

Rietveld H M 1969 J. Appl. Crystallogr. 265

Stokes A R 1948 Proc. Phys. Soc. London 61382

Van Berkum J G M 1994 Strain fields in crystalline of materials, Ph.D. Thesis, Delft University of Technology, The Netherlands

Van Berkum J G M et al 1995 Powder Diffrac. 10129

Wagner C N J 1956 Symp. AIME (New York: Gordon and Breach)

Warren B E 1969 X-ray diffraction (Reading, Mass: AddisonWesley) p. 251

Warren B E and Averbach B L 1950 J. Appl. Phys. 21595

Warren B E and Averbach B L 1952 J. Appl. Phys. 23497 